\title{
Renal artery ablation instead of pulmonary vein ablation in a hypertensive patient with symptomatic, drug-resistant, persistent atrial fibrillation
}

\author{
Dirk Vollmann • Samuel Sossalla • Marco R. Schroeter \\ - Markus Zabel
}

Received: 25 September 2012/ Accepted: 30 November 2012/Published online: 14 December 2012

(C) The Author(s) 2012. This article is published with open access at Springerlink.com

\section{Sirs:}

A 58-year-old female with a history of paroxysmal atrial fibrillation (AF) and essential arterial hypertension (HTN) presented to an external institution with palpitations and progressive symptoms and signs of cardiac decompensation. She had been hospitalized twice for episodes of AF and HTN within the last 2 years and was on a daily medication with metoprolol (95 mg), valsartan (320 mg), hydrochlorothiazide $(25 \mathrm{mg})$, minoxidil $(10 \mathrm{mg})$, and aspirin (100 mg). On admission, blood pressure was $165 / 100 \mathrm{mmHg}$, and surface ECG confirmed recurrence of AF with an irregular ventricular rate of 130-150 bpm. Rate control was pursued by up-titration of $\beta$-blocker dosage and additional administration of digoxin, and therapeutic anticoagulation was initiated. Recompensation was rapidly achieved giving intravenous loop diuretics. Subsequent echocardiography revealed mild left atrial (LA) dilatation, mild mitral regurgitation, and normal systolic but impaired diastolic left ventricular function. While the exact duration of AF was not known, electrical cardioversion was attempted after exclusion of LA thrombi by transesophageal echocardiography. Stable sinus rhythm, however, could not be achieved, even though the patient underwent another electrical cardioversion attempt after administration of amiodarone ( $8 \mathrm{~g}$ in 8 days). Anticoagulation was continued and the patient was referred to our institution for pulmonary vein ablation.

When the patient presented to our center, there were no clinical signs of heart failure but persisting dyspnoea and

D. Vollmann $(\bowtie) \cdot$ S. Sossalla $\cdot$ M. R. Schroeter $\cdot$ M. Zabel Abteilung Kardiologie und Pneumologie,

Georg-August-Universität Göttingen, Universitätsmedizin,

Robert-Koch-Straße 40, 37075 Göttingen, Germany

e-mail: d.vollmann@med.uni-goettingen.de atypical angina upon mild exertion. Blood pressure was $160 / 90 \mathrm{mmHg}$ despite a daily medication with metoprolol (190 mg), valsartan (320 mg), minoxidil (10 mg), spironolacton $(25 \mathrm{mg})$ and furosemide $(60 \mathrm{mg})$. Blood pressures at home (self measurements in the sitting position) had varied between 140 and $160 \mathrm{mmHg}$ (systolic) and 90 and $110 \mathrm{mmHg}$ (diastolic). Serum electrolytes and estimated glomerular filtration rate (eGFR) were within normal ranges. The surface ECG showed that the patient was still in AF, now with a resting ventricular rate of $85 \mathrm{bpm}$. Twenty-four hour Holter monitoring showed persisting AF with a ventricular rate ranging between 79 and $126 \mathrm{bpm}$. Echocardiography was repeated and confirmed the previous findings, with additional evidence for significant pulmonary hypertension [estimated systolic pulmonary artery pressure (PAP) $\sim 70 \mathrm{mmHg}$. Cardiac catheterization revealed post-capillary pulmonary hypertension (PAP systolic $76 \mathrm{mmHg}$, PAP diastolic $40 \mathrm{mmHg}$, PAP mean 53; pulmonary capillary wedge pressure $37 \mathrm{mmHg}$, left ventricular end-diastolic pressure $34 \mathrm{mmHg}$ ) and markedly elevated systemic vascular resistance $\left(4,822 \mathrm{dyn} \times \mathrm{s} \times \mathrm{m}^{2} / \mathrm{cm}^{5}\right.$, normal range 1,970-2,390 dyn $\left.\times \mathrm{s} \times \mathrm{m}^{2} / \mathrm{cm}^{5}\right)$. Angiography excluded significant coronary artery disease. Findings were interpreted as diastolic dysfunction on the basis of chronic hypertension and associated hypertensive heart disease. We discussed all findings with the patient, postponed the LA ablation procedure and recommended ablation in the renal arteries for renal denervation as an initial step for intensified treatment of HTN and potential improvement of her AF. Upon written informed consent, a total of 15 radiofrequency ablation lesions (max. $8 \mathrm{~W}$ for $120 \mathrm{~s}$, respectively) were applied in the right (eight locations, see Fig. 1) and left (seven locations) renal artery using a Symplicity ${ }^{\circledR}$ Catheter (Medtronic/Ardian Inc., USA). No peri-interventional complications occurred, and the patient was discharged in sinus rhythm after AF had 


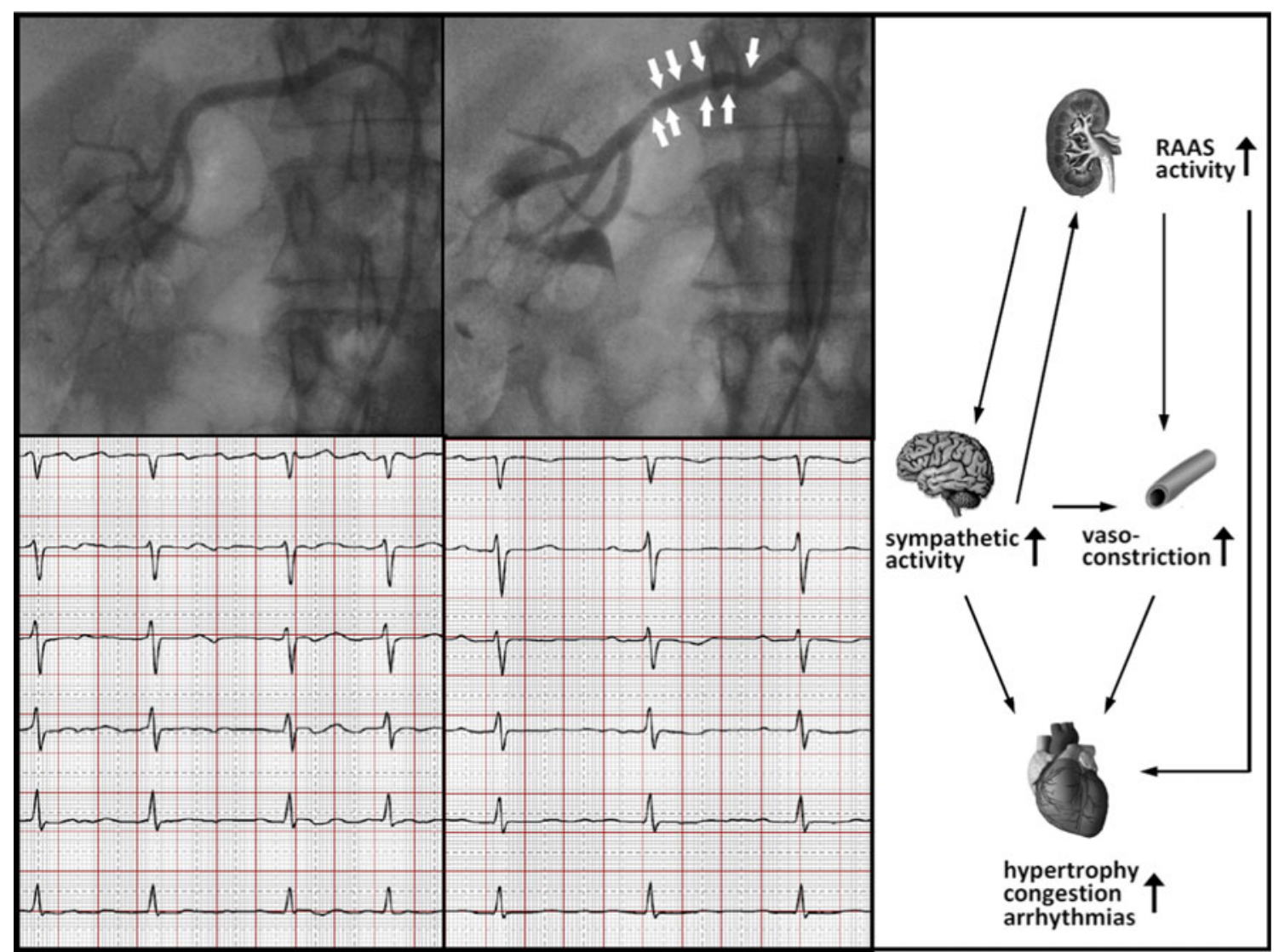

Fig. 1 Top angiographic imaging of the right renal artery before (left) and immediately after (middle) application radiofrequency ablation lesions for renal denervation (arrows indicate presumed local artery constriction/edema in response to thermal application). Bottom surface electrocardiogram (leads $\mathrm{V}_{1}-\mathrm{V}_{6}$ ) showing coarse atrial fibrillation (left) before renal denervation and stable sinus rhythm (right) during follow-up. Right schematic diagram illustrating the proposed relationship between renal afferent nerve activity, central sympathetic tone, peripheral vascular resistance and cardiac arrhythmias in arterial hypertension (from [13] with modification) converted spontaneously (under ongoing amiodarone medication).

Three months later, the patient presented to our outpatient clinic and reported marked improvement in symptoms and exercise capacity. She had no more dyspnoea or angina upon exertion and did not report any palpitations. Amiodarone had been stopped 6 weeks earlier because of transient coughing, whereas antihypertensive medication had not been altered. Blood pressure at presentation was 145/80 mmHg. Ninety-six hour Holter monitoring confirmed stable sinus rhythm with a heart rate of 59-102 bpm. Echocardiographic findings were stable except for a significant decrease in estimated systolic PAP (26 $\mathrm{mmHg}$ as compared to $70 \mathrm{mmHg} 3$ months earlier). Digoxin medication was stopped and furosemide was replaced by hydrochlorothiazide ( $25 \mathrm{mg}$ daily). Another 3 months later the patient was still free of symptoms and in normal sinus rhythm. Ambulatory blood pressure during $24 \mathrm{~h}$ Holter monitoring was $111 / 60 \mathrm{mmHg}$ on average, with a maximum systolic value of $148 \mathrm{mmHg}$ and a maximum diastolic value of $81 \mathrm{mmHg}$. Blood testing indicated that renal function had remained normal after the ablation procedure (eGFR $84 \mathrm{ml} / \mathrm{min}$ ), whereas microalbuminuria was diagnosed before renal denervation but excluded during follow-up. Four days of ECG holter monitoring 5 months after amiodarone medication had been stopped confirmed stable sinus rhythm and ruled out asymptomatic AF recurrences. Atrial ectopy had decreased to an average of $2 / \mathrm{h}$ as compared to $26 / \mathrm{h} 3$ months earlier. Along with these findings, echocardiography showed a progressive decrease of the left atrial diameter from $45 \mathrm{~mm}$ just prior to renal ablation to 40 and $36 \mathrm{~mm} 3$ and 6 months thereafter.

Individual selection of the most appropriate therapy for persistent AF can be cumbersome in subjects that present with heart failure symptoms and cardiovascular co-morbidity. As in the present case, attempts to restore and preserve sinus rhythm appear justified if AF is not longstanding (duration $<1$ year), if the LA is not significantly enlarged, and if symptoms persist despite sufficient rate 
control and in the absence of severe structural heart disease.

In our patient, rhythm control was pursued, and in line with current guidelines [1], pulmonary vein ablation was indicated after antiarrhythmic drug treatment (with amiodarone) had failed to maintain sinus rhythm. Despite the given indication, however, it is well appreciated that the success rate of LA ablation is only moderate in persistent $\mathrm{AF}[1,2]$. In fact, repeated ablation is often required and may in some cases not be restricted to the elimination of triggers (e.g., pulmonary vein isolation) but may also require modification of the perpetuating atrial substrate (e.g., creation of lines of block, targeting sites with complex and fractionated electrograms) [2]. Hypertension and LA dilatation have been identified as strong independent predictors of unsuccessful AF ablation [3]. One study found that LA stiffness, a parameter related to left ventricular diastolic dysfunction, independently predicted unsuccessful AF ablation, whereas LA diameter or LA volume index did not [4].

Catheter ablation in the renal arteries (renal sympathetic denervation) has evolved as a novel therapeutic option for drug-resistant HTN [5, 6]. Its beneficial effects on sympathetic nerve activity [7], the renin-angiotensin-aldosterone system [8], cardiac afterload and left ventricular diastolic dysfunction [9] have also inspired recent interest in its potential impact on $\operatorname{AF}[10,11]$. Of note, a very recent randomized clinical study performed in subjects with drug-resistant HTN and symptomatic AF demonstrated significantly higher success rates of pulmonary vein ablation after 12 months if the procedure was combined with renal artery denervation [12]. The present case is in line with this study and supports the notion that HTN and its associated changes and sequelae are not only an important substrate but also a hitherto underestimated therapeutic target for persistent AF. Furthermore, it has been speculated that renal artery ablation may have antiarrhythmic effects beyond those that result from a pure reduction of cardiac afterload (Fig. 1). Recent interest has focused on the role of excessive central sympathetic drive in chronic disease and on the effects of its reduction by renal denervation [13]. Specifically, reduction of systemic sympathetic activity has also been proposed as an antiarrhythmic mechanism, by which renal ablation may suppress atrial fibrillation $[10,12]$ and refractory ventricular tachyarrhythmias [14]. Although the patient in our present case was on high-dose $\beta$-blocker medication, one can not exclude that reduction of sympathetic activity also had direct effects on atrial electrophysiology (e.g., reduction of atrial ectopy).

In summary, this case illustrates that in patients presenting with 'symptomatic, drug-resistant' AF, careful evaluation and optimized treatment of the underlying substrate (e.g., HTN) should precede consideration for invasive LA ablation procedures. Whether renal denervation in patients with drug-refractory hypertension and atrial fibrillation has an antiarrhythmic effect beyond normalization of blood pressure and cardiac hemodynamics (e.g., due to a reduction of sympathetic activity) remains to be determined.

Open Access This article is distributed under the terms of the Creative Commons Attribution License which permits any use, distribution, and reproduction in any medium, provided the original author(s) and the source are credited.

\section{References}

1. Calkins H, Kuck KH, Cappato R, Brugada J, Camm AJ, Chen SA, et al. (2012) HRS/EHRA/ECAS expert consensus statement on catheter and surgical ablation of atrial fibrillation: recommendations for patient selection, procedural techniques, patient management and follow-up, definitions, endpoints, and research trial design: a report of the Heart Rhythm Society (HRS) task force on catheter and surgical ablation of atrial fibrillation. Developed in partnership with the European heart rhythm association (EHRA), a registered branch of the European society of cardiology (ESC) and the European cardiac arrhythmia society (ECAS); and in collaboration with the American college of cardiology (ACC), American heart association (AHA), the Asia pacific heart rhythm society (APHRS), and the society of thoracic surgeons (STS). Endorsed by the governing bodies of the American college of cardiology foundation, the American heart association, the European cardiac arrhythmia society, the European heart rhythm association, the society of thoracic surgeons, the Asia pacific heart rhythm society, and the heart rhythm society. Heart Rhythm;9(4):632-696 e21

2. Nault I, Miyazaki S, Forclaz A, Wright M, Jadidi A, Jais P et al (2010) Drugs vs. ablation for the treatment of atrial fibrillation: the evidence supporting catheter ablation. Eur Heart $\mathbf{J}$ 31(9): 1046-1054

3. Arya A, Hindricks G, Sommer P, Huo Y, Bollmann A, Gaspar T et al (2010) Long-term results and the predictors of outcome of catheter ablation of atrial fibrillation using steerable sheath catheter navigation after single procedure in 674 patients. Europace 12(2):173-180

4. Machino-Ohtsuka T, Seo Y, Tada H, Ishizu T, Machino T, Yamasaki $H$ et al (2011) Left atrial stiffness relates to left ventricular diastolic dysfunction and recurrence after pulmonary vein isolation for atrial fibrillation. J Cardiovasc Electrophysiol 22(9):999-1006

5. Esler MD, Krum H, Sobotka PA, Schlaich MP, Schmieder RE, Bohm M (2010) Renal sympathetic denervation in patients with treatment-resistant hypertension (the symplicity HTN-2 trial): a randomised controlled trial. Lancet 376(9756):1903-1909

6. Krum H, Schlaich M, Whitbourn R, Sobotka PA, Sadowski J, Bartus $\mathrm{K}$ et al (2009) Catheter-based renal sympathetic denervation for resistant hypertension: a multicentre safety and proofof-principle cohort study. Lancet 373(9671):1275-1281

7. Schlaich MP, Sobotka PA, Krum H, Lambert E, Esler MD (2009) Renal sympathetic-nerve ablation for uncontrolled hypertension. N Engl J Med 361(9):932-934

8. Burke GM, Sica DA, Frishman WH (2012) Renal sympathetic denervation for the treatment of systemic hypertension. Cardiol Rev 20(6):274-278. doi:10.1097/CRD.0b013e3182651f91 
9. Brandt MC, Mahfoud F, Reda S, Schirmer SH, Erdmann E, Bohm $M$ et al (2012) Renal sympathetic denervation reduces left ventricular hypertrophy and improves cardiac function in patients with resistant hypertension. J Am Coll Cardiol 59(10):901-909

10. Linz D, Mahfoud F, Schotten U, Ukena C, Neuberger HR, Wirth $\mathrm{K}$ et al (2012) Renal sympathetic denervation suppresses postapneic blood pressure rises and atrial fibrillation in a model for sleep apnea. Hypertension 60(1):172-178

11. Zhao Q, Yu S, Zou M, Dai Z, Wang X, Xiao J et al (2012) Effect of renal sympathetic denervation on the inducibility of atrial fibrillation during rapid atrial pacing. J Interv Card Electrophysiol 35(2):119-125. doi:10.1007/s10840-012-9717-y
12. Pokushalov E, Romanov A, Corbucci G, Artyomenko S, Baranova V, Turov A et al (2012) A randomized comparison of pulmonary vein isolation with versus without concomitant renal artery denervation in patients with refractory symptomatic atrial fibrillation and resistant hypertension. J Am Coll Cardiol 60(13):1163-1170

13. Sobotka PA, Mahfoud F, Schlaich MP, Hoppe UC, Bohm M, Krum H (2011) Sympatho-renal axis in chronic disease. Clin Res Cardiol 100(12):1049-1057

14. Ukena C, Bauer A, Mahfoud F, Schreieck J, Neuberger HR, Eick C et al (2012) Renal sympathetic denervation for treatment of electrical storm: first-in-man experience. Clin Res Cardiol 101(1):63-67 